\title{
Risk factors for post-transplant lymphoproliferative disorder after pediatric liver transplantation
}

\author{
Limin liu ${ }^{1}$, Kaihua Yang 2 , Tao Zhou ${ }^{3}$, Zhaohui Deng², and Guangxiang $\mathrm{Gu}^{3}$ \\ ${ }^{1}$ Shanghai Jiao Tong University \\ ${ }^{2}$ Shanghai Childrens Medical Center Affiliated to Shanghai Jiaotong University School of \\ Medicine \\ ${ }^{3}$ Shanghai Jiao Tong University School of Medicine Affiliated Renji Hospital
}

August 12, 2020

\begin{abstract}
Abstract Background: Post-transplant lymphoproliferative disorder (PTLD) is a dangerous complication of liver transplantation. This study aimed to analyze the risk factors associated with PTLD after liver transplantation in children. Method: We retrospectively analyzed collected clinical and laboratory data of patients treated and followed-up at Shanghai Children's Medical Center between January 2012 and January 2019. Twenty-four patients with PTLD were enrolled in this study using a 1:2 pairing design. Each case was matched with two controls who had undergone liver transplantation within the same year and did not develop PTLD during the follow-up period. In total, 72 patients were included in this study. Result: No differences in age, gender, weight, primary disease, or type of liver transplantation were observed between those with and without PTLD. Graft weight, graft body weight ratio, transplant type, intraoperative blood loss, blood type, and CMV/EBV infection status of donors and recipients were not related to the occurrence of PTLD. Univariate analysis demonstrated statistically significant differences in EBV infection, tacrolimus blood concentration, PTL, AST, ALT, and CHOL between those with and without PTLD. Multivariate logistics regression analysis demonstrated that EBV infection was an independent risk factor for PTLD. A close relationship was observed between EBV-DNA peak time after transplantation and PTLD onset time. Additionally, the higher the tacrolimus blood concentration, the more difficult it was to control EBV infection. Conclusion: EBV infection is an independent risk factor for PTLD. When uncontrolled proliferation of EBV occurs after organ transplantation, the dosage of immunosuppressive agents should be appropriately reduced
\end{abstract}

\section{Introduction}

With the advancement in medical standards in recent years, children suffering from end-stage liver diseases, such as intrahepatic and extrahepatic cholestasis, metabolic diseases, acute liver failure, and liver tumors, can now undergo liver transplantation. Currently, liver transplantation is the only effective way to treat end-stage liver disease in children; highly-developed surgical techniques and the administration of immunesuppressants have significantly improved the overall survival rate of pediatric patients undergoing liver transplantation. The 5-year survival rate of pediatric patients undergoing liver transplantation in developed countries is as high as $80 \%{ }^{1}$. PTLD is a fatal complication that can occur in children following liver transplantation. Recently, the number of reported PTLD cases has generally increased. PTLD has various clinical manifestations and is difficult to identify at an early stage. The mortality rate of patients is as high as 30\%-60\%; thus, most patients have a poor prognosis. The incidence of PTLD after liver transplantation in children approximately ranges between $1 \%$ and $5 \%^{2,3,4}$, and PTLD is more frequently encountered in pediatric patients with a poor prognosis ${ }^{5}$.

Epstein-Barr virus (EBV) infection is a reported risk factor associated with PTLD. EBV is one of the most 
frequently observed viral pathogens affecting pediatric patients undergoing liver transplant. According to previous reports, EBV infection is closely related to the incidence of PTLD ${ }^{6}$. Similarly, studies have shown that tacrolimus is one of the risk factors in PTLD. The application of tacrolimus after transplantation is known to increase the incidence of malignant tumors and PTLD in patients ${ }^{7,8,9}$; thus, the use of tacrolimus is an additional risk factor associated with PTLD in pediatric patients undergoing a liver transplant. Similarly, patient age at transplantation (between 1-10 years old) and the occurrence of acute rejection after transplantation are related to the occurrence of $\mathrm{PTLD}^{10,11}$.

The risk factors associated with PTLD after liver transplantation in European and North American pediatric patients have been reported in the literature. However, a similar study is currently lacking in the Chinese pediatric population. Here, we conducted a single-center retrospective study to investigate the risk factors of PTLD after liver transplantation in China.

\section{Methods}

\section{Patients}

All patients were treated and followed-up at Shanghai Children's Medical Center. The medical records of patients aged 1-2 years who underwent liver transplantation between January 2012 and January 2019 were reviewed. All patients included in this study were diagnosed with PTLD during the aforementioned period. Patients who died during the follow-up period were excluded from this analysis. The control patients were matched 1:2 according to the type of organ transplanted and the period since transplantation (within 1 year). The control patients were recruited from a cohort of transplant recipients who did not have PTLD and who underwent a transplant during the same period as the study subjects. Clinical characteristics and laboratory findings were extracted from each patient's medical records and analyzed.

\section{Data extraction}

All data sources were extracted from the hospital's medical record management system. Keywords, including "lymphocyte proliferative diseases after transplantation" and "liver transplantation", were used to search the database. The collected indicators included: the (1) basic information: gender, primary disease, age at transplantation, and weight, among others; (2) laboratory indicators: blood test (WBC, HBG, NE, PLT), liver and kidney function (ALT, AST, TBIL, DBIL, ALB, AKP, $\gamma$-GGT, CREA, BUN), blood glucose (GLU), coagulation function (PT, INR), blood lipids (TG, CHOL), and tacrolimus concentration; (3) transplantation-related indicators: transplantation method, graft weight, graft/weight ratio, intraoperative blood loss, and blood type; (4) viral infection: pre-operative EBV and cytomegalovirus (CMV) infections, time interval between surgery, and the first EBV/CMV infection; (5) donor information: blood type, preoperative EBV, and CMV infection, among others.

\section{Treatment}

Data were extracted from hospital medical records, and demographic, clinical, treatment, and laboratory data were obtained. All patients who underwent liver transplantation were administered an anti-immune rejection regimen of tacrolimus and hormones on the first day after surgery. The initial dose of tacrolimus was $0.1-0.15 \mathrm{mg} / \mathrm{kg} / \mathrm{d}$, and the ideal blood concentrations were as follows: 1 month after transplantation, the serum concentration of tacrolimus should be maintained within $8-12 \mathrm{ng} / \mathrm{mL}$; from 2-6 months post-operative, the serum concentration of tacrolimus should be maintained between $7-10 \mathrm{ng} / \mathrm{mL}$ before being reduced to $5-8$ $\mathrm{ng} / \mathrm{mL}$ at $7-12$ months and maintained at $5 \mathrm{ng} / \mathrm{mL}$, as appropriate, 12 months after transplantation. During this period, the drug dosage can be adjusted according to the tacrolimus blood drug concentration and EBV copy number. The total duration of hormone treatment was short. The first dose of glucocorticoid was intravenously administered during the non-hepatic stages of surgery. The dose of intravenous glucocorticoid was reduced to $4 \mathrm{mg} / \mathrm{kg} / \mathrm{d}$ within 1 week post-operative and oral glucocorticoids (e.g. prednisone, an initial dose of $0.25-1 \mathrm{mg} / \mathrm{kg} / \mathrm{d}$ ) were administered 1 week after surgery. Patients with no abnormalities should stop glucocorticoid therapy at the earliest possible opportunity within 3-6 months after surgery.

\section{Follow up}


The outpatient follow-up plan mainly included laboratory tests and imaging examinations, which were designed to monitor graft morphology and graft blood flow. If the patient suffered from unexplained weight loss, fever, gastrointestinal bleeding, anemia, thrombocytopenia, hypoproteinemia, or lymphadenopathy during follow-up, they were informed of the possibility of PTLD. Examinations, such as virological monitoring (EBV and CMV), abdominal and lymph node B-ultrasound, gastrointestinal endoscopy, and positron emission computed tomography (PET-CT), were selected according to varying clinical manifestations of the condition. Imaging examination revealed that patients with obvious abnormal lesions could undergo surgery or gastrointestinal endoscopy to obtain biopsies, which can subsequently facilitate efficient and targeted treatment options based on different pathological manifestations.

\section{Statistical analysis}

Potential risk factors for PTLD were determined by comparing different variables between patients with PTLD and control subjects using SPSS Software. The primary analyses were conducted by the T-test and Chi-square test. To eliminate confounding factors affecting the results, multiple logistic regression was used to further analyze the results. A $\mathrm{P}$ value $<0.05$ was considered statistically significant.

\section{Results}

\section{Baseline characteristics}

After screening, according to the inclusion and exclusion criteria, a total of 24 patients in the PTLD group were included. Furthermore, a total of 48 control patients were enrolled.

The descriptive characteristics of the study subjects are shown in Table 1. Males and females comprised $33.3 \%$ and $66.7 \%$ of the PTLD group, respectively. On the contrary, males and females comprised $37.5 \%$ and $62.5 \%$ of the control group, respectively. The average age at transplantation for patients with PTLD was 9.96 months: the minimum age was five months, and the maximum age was 24 months. On the contrary, the average age at transplantation in the control group was 8.60 months: the minimum age was five months, and the maximum age was 24 months. No significant difference in the distribution of transplantation age and sex was observed between the two groups. Among the included cases, the primary disease affecting the two groups was biliary atresia.

\section{Laboratory indicators and liver transplantation characteristics}

Analysis of preoperative laboratory indicators (Table 2), including blood tests (WBC, HBG, NE, PLT), liver and kidney functions (ALT, AST, TBIL, DBIL, ALB, AKP, $\gamma$-GGT, BUN, CREA), blood glucose (GLU), coagulation function (PT, INR), and blood lipids (TG, CHOL) between the PTLD group and the control group revealed that there were statistically significant differences in postoperative platelets, ALT, AST, CHOL and serum tacrolimus concentration $(\mathrm{P}<0.05)$. The patient was administered tacrolimus (anti-rejection treatment plan) after liver transplantation; therefore, their serum tacrolimus concentrations were continuously monitored. Statistical analysis revealed that the average serum tacrolimus concentrations of patients in the PTLD and control groups were $8.28 \pm 1.61 \mathrm{ng} / \mathrm{mL}$ and $7.32 \pm 1.63 \mathrm{ng} / \mathrm{mL}$, respectively, postoperative. Similarly, a statistically significant difference was observed between the serum tacrolimus concentrations of the PTLD and control groups $(\mathrm{P}<0.05)$ (Table 2).

As shown in Table 2, graft weight, graft body weight ratio, transplant type, intraoperative blood loss, and blood type were not related to the occurrence of PTLD.

\section{Viral infection}

The EBV and CMV infection status of patients and donors was analyzed. It was observed that the PTLD group had an EBV infection rate of $95.8 \%$ and a CMV infection rate of $20.8 \%$, which differed from the EBV and CMV infection rates of the control group, which were $48.9 \%$ and $22.9 \%$, respectively. A significant difference in EBV infection rates was observed between the two groups. Statistical analysis demonstrated that EBV infection is an important risk factor for PTLD $(\mathrm{P}<0.001)$. No association was observed between CMV 
and PTLD in this study. The EBV and CMV infection statuses of donors before transplantation were classified as follows: donor-positive/recipient-negative, donor-positive/recipient-positive, donor-negative/recipientpositive, and donor-negative/recipient-negative. Statistical analysis showed that the viral infection status of donors and recipients before surgery were not statistically associated $(\mathrm{P}>0.05)$ (Table 3$)$.

\section{Risk factor for PTLD after liver transplantation}

Considering the influence of confounding factors on the results of single-factor analysis, factors, such as EBV infection, tacrolimus concentration, ALT, AST, PLT, and CHOL, should be further analyzed by multivariate logistic regression analysis.

The results showed that EBV infection was an independent risk factor for PTLD $(\mathrm{P}<0.05)$. Tacrolimus concentration, ALT, AST, PLT, and CHOL had varying degrees of difference in the incidence of PTLD; however, no statistical differences were identified $(\mathrm{P}>0.05)$ (Table 4).

The relationship between EBV-DNA peak time, PTLD onset time, and tacrolimus concentration in the PTLD group

In the PTLD group, a close relationship was observed between EBV-DNA peak time after transplantation and PTLD onset time $(|\mathrm{r}|=0.873, \mathrm{P}<0.01)$; this suggests that PTLD is prone to occur during uncontrolled proliferation of EBV infection. (Table 5).

Analysis of the time between peak EBV-DNA and peak tacrolimus concentration suggested that the higher the blood concentration of tacrolimus, the more difficult it was to control EBV infection $(|\mathrm{r}|=-0.481, \mathrm{P}<0.05)$. (Table 6).

\section{Discussion}

Many studies have confirmed that EBV infection is directly related to the incidence of PTLD ${ }^{12,13}$. This fact was confirmed by the findings of this study. The EBV infection rate of the 24 PTLD patients included in the analysis was $95.8 \%$. The analysis suggested that EBV infection was an important risk factor for PTLD. In the PTLD group, the time of peak EBV copying was closely related to the time of PTLD onset, which indicates that PTLD is likely to occur when the EBV infection replicates rapidly and reaches its peak. Therefore, monitoring changes in the EBV copy number in transplant patients will allow clinicians to monitor PTLD and appropriately adjust the level of immunosuppressants administered to patients. Furthermore, changes in the EBV copy number may prompt the use of rituximab therapy ${ }^{14}$. Recent research observed that the first-time application of rituximab could be performed in patients whose EBV-DNA load is $>40000 \mathrm{U} / \mathrm{mL}$ after transplantation if they similarly exhibited at least one symptom of PTLD or demonstrated evidence of PTLD upon imaging ${ }^{15}$.

CMV infection is common after liver transplantation in children and is associated with the following symptoms: fever of unknown origin $\left(38.3^{\circ} \mathrm{C}\right)$, fatigue or systemic myalgia, and leukopenia or thrombocytopenia. The relationship between CMV and PTLD remains unclear. Some studies believe that CMV infection is a risk factor of PTLD, although the mechanism of action is currently unknown. Most studies believe that CMV infections are not related to the occurrence of PTLD. JM, Kim ${ }^{16}$ analyzed 119 liver transplant patients. Among them, $66(55.5 \%)$ patients had a CMV infection, and 15 of 119 patients eventually developed PTLD. Among them, 10 patients with PTLD had a CMV infection, whereas 5 patients had no CMV infection. Statistical analysis showed that the occurrence of PTLD was not related to CMV infection $(\mathrm{P}=0.258)$. In this study, the CMV infection rate was $22.2 \%$, and the final incidence of PTLD in CMV-infected patients was $31.2 \%$. No statistically significant relationship was observed between CMV infection and PTLD ( $\mathrm{P}=1.000)$.

Studies have shown that donor $\mathrm{EBV}^{+} /$recipient $\mathrm{EBV}^{-}$is an important risk factor for PTLD as a high EBV infection rate can cause PTLD in younger patients. Previous research has found that in donor $\mathrm{EBV}^{+} / \mathrm{recipient}$ $\mathrm{EBV}^{-}$patients, $67 \%$ of patients develop a postoperative EBV infection. A higher postoperative EBV infection rate increases the risk of PTLD in patients. EBV-negative patients receiving EBV-positive organs are at high risk of developing PTLD ${ }^{17}$. This study assessed the viral infection status of the donors. Seven of the study 
patients received cadaveric liver transplants; thus, in these cases, the infection status of the donor could not be obtained. Only 65 patients who underwent liver transplants were analyzed, and the virus infection rate of the donor was not high. Subsequently, no statistical significance was identified; this may be due to the sample size.

Some studies have suggested that a high serum concentration of tacrolimus is a risk factor for the onset of PTLD ${ }^{18}$. Daizo Fukushima ${ }^{19}$ analyzed the changes in tacrolimus concentrations when the EBV load continued to increase and observed that the tacrolimus concentrations in the PTLD group were significantly higher than those in the control group. Other research has focused on the correlation between tacrolimus and EBV infection. By monitoring serum tacrolimus concentrations within 2 weeks of liver transplantation, $\mathrm{Lu}^{20}$ found that the EBV infection rate increased within 2-4 weeks post-operatively in patients with a high serum concentration of tacrolimus. Bakker ${ }^{21}$ observed that when the immunosuppressant dosages were reduced in patients suffering from uncontrolled, post-operative EBV infections, the EBV load significantly reduced.

This study evaluated the relationship between serum tacrolimus concentrations, PTLD, and EBV. Univariate analysis of the PTLD group and the control group showed a statistical difference in the serum concentrations of tacrolimus $(\mathrm{P}=0.021)$; however, multivariate analysis demonstrated that serum tacrolimus concentration levels were not a risk factor for PTLD, although the serum tacrolimus concentrations of patients in the PTLD group were higher than those of the control group. However, when analyzing the relationship between the peak time of EBV infection and the serum tacrolimus concentrations of patients in the PTLD group, it was observed that at a higher level of immunosuppression, EBV is prone to uncontrolled proliferation. This is because tacrolimus acts upon both B lymphocytes and T lymphocytes while suppressing humoral and cellular immunity. This subsequently leaves patients prone to EBV infections after surgery and makes it difficult to control EBV proliferation after infection ${ }^{22,23}$. In view of the fact that EBV infection is a risk factor for PTLD, it is suggested that when an uncontrolled proliferation of EBV occurs after organ transplantation, the amount of immunosuppressive agents should be appropriately reduced to prevent PTLD.

In this study, no association was observed between baseline characteristics, laboratory indicators, and transplantation-related indicators and the occurrence of PTLD. The current controversy is the relationship between the patient's age at the time of transplantation and PTLD. A study observed that younger patients undergoing organ transplantation are at a greater risk of PTLD, especially children (1-10 years old $)^{24}$; Other research concluded that the risk of postoperative PTLD reduces by $15 \%$ with a yearly increase in age ${ }^{25}$ and found that younger patients (at the time of transplantation) is a risk factor for EBV and CMV after surgery ${ }^{26}$. No association was observed between age at transplantation and the occurrence of PTLD; the possible reason could be that the primary disease of the patients included in this study was mostly biliary atresia, and the transplantation age of patients is generally within 2 years. Young patients are probably more susceptible to PTLD because they have a higher viral infection rate than adults at the time of transplantation.

\section{Conclusion}

PTLD is a fatal complication that occurs after liver transplantation in children. Baseline characteristics, laboratory indicators, and transplantation-related indicators are not related to the occurrence of PTLD. The results of this study suggest that EBV infection is an independent risk factor for PTLD; thus, it is necessary to be aware of the risk of PTLD when EBV is continuously replicating or the viral load is high. In addition, tacrolimus is not a direct factor leading to the occurrence of PTLD; however, a high serum tacrolimus concentration is related to EBV infection. The degree of immunosuppression should be reduced in children with continuously replicating EBV and a high concentration of tacrolimus in the blood.

\section{Conflict of interest statement}

The authors declare no conflict of interest associ- ated with this manuscript.

\section{Acknowledgements}

This work was supported in part by hospital data management center. 


\section{References}

1. Kasahara M, Umeshita K, Inomata Y, et al. Long-term outcomes of pediatric living donor liver transplantation in Japan analysis of more than 2200 cases listed in the registry of the Japanese Liver Transplantation Society[J]. Am J Transplant 2013;13:1830-1839.

2. Lauro A, Arpinati M, Pinna AD. Managing the challenge of PTLD in liver and bowel transplant recipients[J]. Br J Haematol 2014;169:157-172.

3. Kumarasinghe G, Lavee O, Parker A, et al. Post-transplant lymphoproliferative disease in heart and lung transplantation: Defining risk and prognostic factors[J]. J Heart Lung Transplant 2015;34:1406-1414.

4. Martin M, Tilmann S, Uta B, et al. Posttransplant lymphoproliferative disease after pediatric solid organ transplantation[J]. Clin Dev Immunol 2013;(2013):1-14.

5. Jeong HJ, Ahn YH, Park E, et al. Posttransplantation lymphoproliferative disorder after pediatric solid organ transplantation: Experiences of 20 years in a single center[J]. Korean J Pediatr 2017;60:86-93.

6. Ruf S, Behnke-Hall K, Gruhn B, et al. Comparison of six different specimen types for Epstein-Barr viral load quantification in peripheral blood of pediatric patients after heart transplantation or after allogeneic hematopoietic stem cell transplantation [J]. Clin Virol 2012;53:186-194.?

7. Schubert S, Renner C, Hammer M, et al. Relationship of immunosuppression to Epstein-Barr viral load and lymphoproliferative disease in pediatric heart transplant patients[J]. J Heart Lung Transplant 2008;27:0105.

8. Parker A, Bowles K, Bradley JA, et al. Diagnosis of post-transplant lymphoproliferative disorder in solid organ transplant recipients-BCSH and BTS guidelines. Br J Haematol 2010;149:675-692.

9. Jagadeesh D, Woda BA, Draper J, Evens AM. Posttransplant lymphoproliferative disorders: risk, classification, and therapeutic recommendations. Curr Treat Options in Oncol 2012;13:122-136.

10. Chinnock R, Webber SA, Dipchand AI, et al. A 16-Year multi-institutional study of the role of age and EBV Status on PTLD incidence among pediatric heart transplant recipients[J]. American J Transplant 2012;12:3061-3068.

11. Dolcetti R. B lymphocytes and Epstein-Barr virus: The lesson of post-transplant lymphoproliferative disorders[J]. Autoimmun Rev 2008;7:96-101.

12. Young LS, Rickinson AB. Epstein-Barr virus: 40 years on[J]. Nat Rev Cancer 2004;4:757-768.

13. Kimura H, Ito Y, Suzuki R, Nishiyama Y. Measuring Epstein-Barr virus (EBV) load: the significance and application for each EBV-associated disease [J]. Rev Med Virol 2008;18:305-319.

14. Kalra A,Roessner C,Jupp J,et al. Epstein-bar virus DNA-emia monitoring for the management of posttransplant lymphoproliferative disorder [J]. Cytotherapy 2018;20:706-714.

15. Kim JM, Lee SK, Kim SJ, et al. Risk factors for posttransplant lymphoproliferative disorder in pediatric liver transplant recipients with cytomegalovirus antigenemia[J]. Transplant Proc 2010;42:0-899.

16. Cockfield SM. Identifying the patient at risk for post-transplant lymphoproliferative disorder[J]. Transpl Infect Dis 2001;3:70-78.

17. Jagadeesh D, Woda BA, Draper J, Evens AM. Posttransplant lymphoproliferative disorders: risk, classification, and therapeutic recommendations. Curr Treat Options in Oncol 2012;13:122-136.

18. Fukushima D, Sato K, Kawagishi N, et al. Epstein-Barr virus - associated posttransplantation lymphoproliferative disorder with tacrolimus metabolism deterioration in infants after living-donor liver transplantation[J]. Transplantation 2015;99:114-119. 
19. Lu BR, Park KT, Hurwitz M, et al. Impact of immunosuppression on the development of Epstein-Barr virus (EBV) viremia after pediatric liver transplantation[J]. Transplant Proc 2013;45:301-304.

20. Bakker NA, Verschuuren EAM, Erasmus ME, et al. Epstein-Barr virus-DNA load monitoring late after lung transplantation: a surrogate marker of the degree of immunosuppression and a safe guide to reduce immunosuppression [J]. Transplantation 2007;83:433-438.

21. Cosmas D, Wimmer, Martin K, et al. Impact of cyclosporine versus tacrolimus on the incidence of de novo malignancy following liver transplantation: a single center experience with 609 patients $[\mathrm{J}]$. Transpl Int 2013;26:999-1006.

22. Taylor AL, Marcus R, Bradley JA. Post-transplant lymphoproliferative disorders (PTLD) after solid organ transplantation[J]. Crit Rev Oncol Hematol 2005;56:155-167.

23. Chinnock R, Webber SA, Dipchand AI et al. A 16-Year multi-institutional study of the role of age and EBV Status on PTLD incidence among pediatric heart transplant recipients[J]. Am J Transplant 2012;12:3061-3068.

24. Guthery SL, Heubi JE, Bucuvalas JC, et al. Determination of risk factors for Epstein-Barr virusassociated posttransplant lymphoproliferative disorder in pediatric liver transplant recipients using objective case ascertainment[J]. Transplantation 2003;75:987-993.

25. Shepherd RW, Turmelle Y, Nadler M, et al. Risk factors for rejection and infection in pediatric liver transplantation[J]. Am J Transplant 2008;8:396-403.

\section{Hosted file}

Table 1.docx available at https://authorea.com/users/350353/articles/475232-risk-factorsfor-post-transplant-lymphoproliferative-disorder-after-pediatric-liver-transplantation

\section{Hosted file}

Table 2.docx available at https://authorea.com/users/350353/articles/475232-risk-factorsfor-post-transplant-lymphoproliferative-disorder-after-pediatric-liver-transplantation

\section{Hosted file}

Table 3.docx available at https://authorea.com/users/350353/articles/475232-risk-factorsfor-post-transplant-lymphoproliferative-disorder-after-pediatric-liver-transplantation

\section{Hosted file}

Table 4.docx available at https://authorea.com/users/350353/articles/475232-risk-factorsfor-post-transplant-lymphoproliferative-disorder-after-pediatric-liver-transplantation

\section{Hosted file}

Table 5.docx available at https://authorea.com/users/350353/articles/475232-risk-factorsfor-post-transplant-lymphoproliferative-disorder-after-pediatric-liver-transplantation

\section{Hosted file}

Table 6.docx available at https://authorea.com/users/350353/articles/475232-risk-factorsfor-post-transplant-lymphoproliferative-disorder-after-pediatric-liver-transplantation 